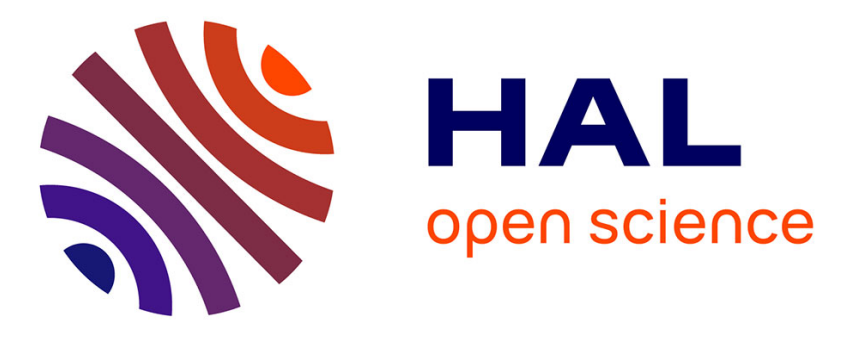

\title{
Generation of recombinant pestiviruses using a full-genome amplification strategy
}

Thomas Bruun Rasmussen, Ilona Reimann, Åse Uttenthal, Immanuel Leifer, Klaus Depner, Horst Schirrmeier, Martin Beer

\section{To cite this version:}

Thomas Bruun Rasmussen, Ilona Reimann, Åse Uttenthal, Immanuel Leifer, Klaus Depner, et al.. Generation of recombinant pestiviruses using a full-genome amplification strategy. Veterinary Microbiology, 2010, 142 (1-2), pp.13. 10.1016/j.vetmic.2009.09.037 . hal-00578396

\section{HAL Id: hal-00578396 https://hal.science/hal-00578396}

Submitted on 20 Mar 2011

HAL is a multi-disciplinary open access archive for the deposit and dissemination of scientific research documents, whether they are published or not. The documents may come from teaching and research institutions in France or abroad, or from public or private research centers.
L'archive ouverte pluridisciplinaire HAL, est destinée au dépôt et à la diffusion de documents scientifiques de niveau recherche, publiés ou non, émanant des établissements d'enseignement et de recherche français ou étrangers, des laboratoires publics ou privés. 


\section{Accepted Manuscript}

Title: Generation of recombinant pestiviruses using a full-genome amplification strategy

Authors: Thomas Bruun Rasmussen, Ilona Reimann, Åse Uttenthal, Immanuel Leifer, Klaus Depner, Horst Schirrmeier, Martin Beer

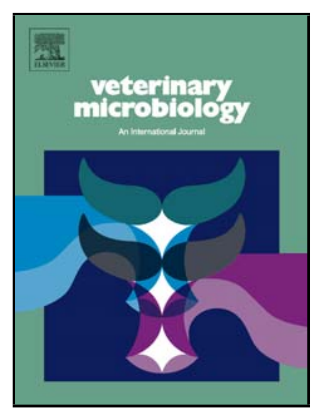

PII: S0378-1135(09)00454-4

DOI: doi:10.1016/j.vetmic.2009.09.037

Reference: VETMIC 4594

To appear in: VETMIC

Please cite this article as: Rasmussen, T.B., Reimann, I., Uttenthal, Å., Leifer, I., Depner, K., Schirrmeier, H., Beer, M., Generation of recombinant pestiviruses using a full-genome amplification strategy, Veterinary Microbiology (2008), doi:10.1016/j.vetmic.2009.09.037

This is a PDF file of an unedited manuscript that has been accepted for publication. As a service to our customers we are providing this early version of the manuscript. The manuscript will undergo copyediting, typesetting, and review of the resulting proof before it is published in its final form. Please note that during the production process errors may be discovered which could affect the content, and all legal disclaimers that apply to the journal pertain. 
Formatted for Veterinary Microbiology (special ESVV Pestivirus issue)

1 Generation of recombinant pestiviruses using a full-genome amplification

2 strategy

3

4 Thomas Bruun Rasmussen ${ }^{1^{*}}$, Ilona Reimann ${ }^{2}$, Åse Uttenthal ${ }^{1}$, Immanuel Leifer $^{2}$,

$5 \quad$ Klaus Depner ${ }^{2}$, Horst Schirrmeier ${ }^{2}$ and Martin Beer ${ }^{2}$

6

$7{ }^{1}$ DTU National Veterinary Institute, Technical University of Denmark, Lindholm,

8 DK-4771 Kalvehave, Denmark

$9{ }^{2}$ Institute of Diagnostic Virology, Friedrich-Loeffler-Institut, D-17493 Greifswald-

10 Insel Riems, Germany

$12{ }^{*}$ Corresponding author

13 Thomas Bruun Rasmussen

14 DTU National Veterinary Institute (DTU Vet)

15 Technical University of Denmark

16 Lindholm

17 DK-4771 Kalvehave

18 Phone : +4535887850

19 Fax : +4535887901

20 Email : tbrur@vet.dtu.dk 
Formatted for Veterinary Microbiology (special ESVV Pestivirus issue)

\section{Abstract}

23 Complete genome amplification of viral RNA provides a new tool for the

24 generation of modified viruses. We have recently reported a full-genome

25 amplification strategy for recovery of pestiviruses (Rasmussen et al., 2008). A

26 full-length cDNA amplicon corresponding to the Border disease virus-Gifhorn

27 genome was generated by long RT-PCR and then RNA transcripts derived from

28 this amplicon were used to rescue infectious virus. Here, we have now used this

29 full-genome amplification strategy for efficient and robust amplification of three

30 additional pestivirus strains: the vaccine strain $\mathrm{C}$ and the virulent Paderborn

31 strain of Classical swine fever virus plus the CP7 strain of Bovine viral diarrhoea

32 virus. The amplicons were cloned directly into a stable single-copy bacterial

33 artificial chromosome generating full-length pestivirus DNAs from which

34 infectious RNA transcripts could be also derived.

36 Keywords: Pestivirus, Flaviviridae, long RT-PCR, full-genome amplicon,

37 infectious clone, pBeloBAC11 
Formatted for Veterinary Microbiology (special ESVV Pestivirus issue)

\section{Introduction}

The genus Pestivirus is part of the family Flaviviridae and contains economically important animal viruses such as Classical swine fever virus (CSFV), Bovine viral diarrhoea virus (BVDV) and Border disease virus (BDV). Pestivirus virions are enveloped and contain a positive-stranded RNA genome of about $12.3 \mathrm{~kb}$ which has a single long open reading frame flanked by non-translated regions (5'NTR and 3'NTR), which are important for viral replication. Generation of pestivirus cDNA clones is a general prerequisite for genetic manipulation of their genomes, a process that can be both lengthy and laborious. Several infectious cDNA clones containing complete pestivirus genomes have been described (Meyers et al., 1996; Moormann et al., 1996; Ruggli et al., 1996; Vassilev et al., 1997; Fan and Bird, 2008). These cDNA clones have provided new options for directed genetic manipulations of pestiviruses. However, construction of these infectious cDNA clones has been hampered due to the relatively large size of the viral RNA genome and because of genetic instability of the cloned cDNA in combination with the plasmid vectors in the bacterial host. Therefore, new strategies are needed to facilitate construction of stable infectious cDNA clones from a wider range of strains.

Generation of infectious cDNA clones can be facilitated by using long RT-PCR for full-genome amplification. This approach has been applied with success to a number of RNA viruses from the Flaviviridae (Tellier et al., 1996; Gritsun and Gould, 1998; Zhang et al., 2001). For pestiviruses, the methodology has been used to obtain the entire open reading frame for sequencing studies (Jones et al., 2006) as well as for virus rescue (Rasmussen 
Formatted for Veterinary Microbiology (special ESVV Pestivirus issue)

et al., 2008). Full-length genome amplification facilitates, in addition to virus rescue, direct full-length sequence analysis and the amplicons can also be directly inserted into suitable cloning vectors.

In the present study, we developed an optimized, efficient and streamlined method for the robust amplification of full-length cDNAs of four strains (BVDV-CP7, BDV-Gifhorn, CSFV-C and CSFV-Paderborn). This procedure was followed by the insertion of the BDV and CSFV amplicons into the stable single-copy vector pBeloBAC11 (Wang et al., 1997). This bacterial artificial chromosome (BAC) has been shown to successfully maintain the fulllength cDNAs of Japanese encephalitis virus (Yun et al., 2003) and BVDV-SD1 (Fan and Bird, 2008). Using this procedure, we have generated new full-length cDNA clones of BDV and CSFV, which enlarges the range of cloned pestivirus genomes that can be manipulated.

\section{Materials \& Methods}

The different viruses used in this study were; BVDV-CP7 from bovine KOP-R cells $\left(10^{6} \mathrm{TCID}_{50} / \mathrm{ml}\right)$; CSFV-Paderborn from porcine PK15 cells $\left(10^{7} \mathrm{TCID}_{50} / \mathrm{ml}\right.$; vaccine strain CSFV-C from porcine PK15 cells $\left(10^{4.75} \mathrm{TCID}_{50} / \mathrm{ml}\right)$; and BDVGifhorn from ovine SFT cells $\left(10^{6} \mathrm{TCID}_{50} / \mathrm{ml}\right)$. Infected cells were grown in Dulbecco's Modified Eagle Medium supplemented with 10\% BVDV-free foetal bovine serum at $37^{\circ} \mathrm{C}$ in a humidified atmosphere containing $5 \% \mathrm{CO}_{2}$. The total RNA was extracted from the cells using a combined Trizol/RNeasy protocol. This protocol was used to obtain high-quality full-length genomic RNA. Briefly, total RNA was extracted from $1 \mathrm{ml}$ cell supernatant or cell lysate with $3 \mathrm{ml}$ Trizol 
Formatted for Veterinary Microbiology (special ESVV Pestivirus issue)

87 LS reagent (Invitrogen) and $0.8 \mathrm{ml}$ chloroform. After phase separation the aqueous phase was mixed with an equal volume of $75 \%$ ethanol and $0.7 \mathrm{ml}$ of the resulting mix was transferred sequentially to the same RNeasy column (Qiagen). The column was washed according to the manufacturer's protocol.

91 Total RNA was eluted from the column with 4 times $30 \mu \mathrm{l}$ nuclease free water and stored at $-80^{\circ} \mathrm{C}$ until use. Pestivirus genomes were amplified from total RNA preparations using long RT-PCR. It was found that the third RNA eluate empirically performed the best in long RT-PCR, presumable due to a higher proportion of full-length genomic RNA in this fraction. The total RNA was reverse transcribed at $50^{\circ} \mathrm{C}$ for 90 minutes using SuperScript III reverse transcriptase kit (Invitrogen) and specific cDNA primers (Table 1). For improved long RT-PCR efficiency, the resulting cDNA was subsequently treated with RNAse $\mathrm{H}$ to remove the RNA template. Full-

100 length PCR amplification was performed using primers specific for the 5'NTR and the 3'NTR of each strain (Table 1) with the Accuprime High Fidelity kit

102 (Invitrogen) which consists of a mixture of Taq and proofreading Pyrococcus GB-D DNA polymerases. Reactions containing $2 \mu \mathrm{l}$ cDNA were then amplified

104 in a final volume of $50 \mu \mathrm{l}$ using $94^{\circ} \mathrm{C}$ for 30 seconds followed by 35 cycles of $94^{\circ} \mathrm{C}$ for 15 seconds, $65^{\circ} \mathrm{C}$ for 30 seconds and $68^{\circ} \mathrm{C}$ for 12 minutes. Samples $(1-10 \mu l)$ of each PCR reaction were analysed on a $1 \%$ agarose gel in TBE

107 buffer alongside a 1kb DNA ladder (Fermentas). amplicons into pBeloBAC11 (Invitrogen). Briefly, pBeloBAC11 (7507 bp) was

110 digested with Notl and afterwards treated with Antarctic phosphatase (NEB). 
Formatted for Veterinary Microbiology (special ESVV Pestivirus issue)

111 The full-length amplicons of about $12.3 \mathrm{~kb}$ were also digested with Notl and

112 purified using the Nucleotide removal kit (Qiagen). Equal amounts of vector and

113 amplicons were mixed and ligated in $25 \mu \mathrm{l}$ reactions overnight at $16^{\circ} \mathrm{C}$ using

114 DNA ligase for long fragments (Takara Bio) according to the manufacturer's

115 recommendations. The products $(1 \mu \mathrm{l})$ were electroporated into

116 electrocompetent DH10B T1 phage resistant E.coli (Invitrogen) and

117 transformants harbouring the cloned amplicons were isolated from selective LB

118 plates containing $12.5 \mu \mathrm{g} / \mathrm{ml}$ chloramphenicol. Small scale preparations of DNA

119 from selected transformants were obtained using the BAC miniprep protocol

120 previously described (Warming et al., 2005) and the presence of the full-length

121 pestivirus cDNA was determined following restriction analysis using Notl.

122 Selected BAC DNAs were digested with Notl, in vitro transcribed using T7 RNA

123 polymerase and the RNA transcripts were tested for infectivity as previously

124 described (Reimann et al., 2004; Rasmussen et al., 2007). Immunostaining of

125 electroporated porcine SK6 cells (CCLV RIE262) was performed with the pan-

126 pestivirus antibody (C16) targeting the non-structural NS3 proteins (Reimann et

127 al., 2004).

128

\section{Results}

130 Full-length cDNAs corresponding to four different pestivirus strains were

131 successfully amplified in single long RT-PCRs using an optimized version of the

132 full-genome amplification strategy described by Rasmussen et al. (2008). The

133 original protocol was modified by substituting the Elongase enzyme mix with the

134 Accuprime High Fidelity kit. This polymerase mix, in combination with the 
Formatted for Veterinary Microbiology (special ESVV Pestivirus issue)

135 Superscript III reverse transcriptase, efficiently produced, from each of the

136 different viral RNAs, amplicons of approximately $12.3 \mathrm{~kb}$, corresponding to the

137 full-length genomes (Fig. 1). The Accuprime High Fidelity polymerase mix

138 increased the specificity and efficiency of the long RT-PCR compared to the

139 Elongase enzyme mix and produced distinct products of the expected size

140 without smears and aberrant amplification products (Fig. 1). High yields (several

141 micrograms) of DNA were obtained in each long RT-PCR. For amplification of

142 complete genomes in a single RT-PCR, specific primers were designed based

143 on nucleotide sequences of the terminal 5'NTR- and 3'NTR-genomic ends

144 (Table 1). The primers for amplification of BDV-Gifhorn were described in

145 Rasmussen et al. (2008). The primers for BVDV-CP7 (Meyers et al., 1996,

146 Accession number BVU63479), and for the CSFV-C (Accession number

147 AY259122) were based on published sequences. Similarly, the 5'NTR primer

148 for CSFV-Paderborn was designed from Accession number AY072924

149 (Oleksiewicz et al., 2003) but this sequence lacks approximately 75 nucleotides

150 from the terminus of the 3'NTR. Therefore, the 3'Paderborn reverse primer was

151 designed using a nucleotide alignment of known full-genome CSFV sequences.

152 Generally, knowledge of less than 50 nucleotides of the terminal sequences

153 from each of the strains was needed for successful amplification of the whole

154 genome.

By incorporating unique Notl restriction sites within the primers

156 (Table 1), the amplicons were designed for subsequent insertion into

157 pBeloBAC11. Full-length amplicons of the two strains CSFV-C and CSFV-

158 Paderborn, and also the BDV-Gifhorn were obtained using the respective Notl 
Formatted for Veterinary Microbiology (special ESVV Pestivirus issue)

159 primers (Fig. 2). Amplification and cloning of BVDV-CP7 was not attempted by

160 this strategy due to an internal Notl site in the cDNA sequence.

The amplified genomes were digested with Notl and ligated directly

162 into the Notl site in pBeloBAC11. Electroporation of DH10B E.coli cells with the

163 ligated products yielded small and large bacterial colonies (from a few to

164 several hundreds) after incubation for 24 hours at $37^{\circ} \mathrm{C}$. Both small and large

165 colonies were tested for the presence of full-genome amplicons. Generally, two

166 thirds of the tested transformants carried a BAC containing an insert of the

167 expected size of approximately $12.3 \mathrm{~kb}$ (Fig. 3). One third of the transformants

168 either carried BACs with smaller inserts, empty BACs or no visible BAC vector.

169 There was no correlation between the size of the BAC inserts and the size of

170 the bacterial colonies.

Testing of the BAC clones for their ability to generate virus

172 infectivity in cells is ongoing in our laboratories. The preliminary results of this evaluation show that infectious pestiviruses can be rescued from the DNAs

174 generated by direct insertion of full-length genomes into the BAC vector. For example, selected BAC clones harbouring the CSFV-Paderborn full-length

176 CDNA were Notl digested, in vitro transcribed and the RNA transcripts were

177 tested for infectivity by electroporation into porcine cells. RNA derived from one

178 out of two tested BAC clones (pBeloPader10) was shown to efficiently replicate

179 in porcine cells. The cells electroporated with RNA transcripts from

180 pBeloPader10 showed a high number of NS3-positive cells (as judged by

181 immunofluorescence, Fig. 4) 24 hours later and infectious virus could be

182 recovered after $1^{\text {st }}$ and $2^{\text {nd }}$ cell passages. In addition, RNA transcripts derived 
Formatted for Veterinary Microbiology (special ESVV Pestivirus issue)

183 from three BAC clones harbouring BDV-Gifhorn full-length cDNA have been

184 tested for infectivity in ovine cells. Following electroporation, viral RNA derived

185 from each of these three BAC clones was found to be replicating in the cells

186 (data not shown). However, only rather few cells were scored as positive after

187 the $1^{\text {st }}$ cell passage indicating a possible growth defect in these constructs.

189 Discussion

190 The use of the full-length genome amplification strategy for amplification of

191 pestivirus genomes has several advantages; (i) the same optimized protocol

192 can be used for amplification of a wide selection of strains; (ii) only limited

193 terminal nucleotide sequence information of a given strain is needed for

194 amplification of the whole genome; (iii) multiple cloning steps can be

195 circumvented since full-length amplicons can be directly in vitro transcribed or

196 inserted into plasmid vectors; and (iv) construction of genetically modified

197 viruses can be facilitated using stable cDNA clones.

198 In the present study, four different pestiviruses representing each of

199 three main types (CSFV, BVDV and BDV) were amplified by long RT-PCR. This

200 demonstrates that the recently described full-length genome amplification

201 strategy (Rasmussen et al., 2008) is of a generic nature since it can be utilized

202 for amplification of diverse pestivirus strains. Furthermore, the direct cloning of

203 the amplified genomes into the stable single-copy BAC vector, pBeloBAC11,

204 shows that we have created an efficient, streamlined strategy for direct

205 preparation of new pestivirus cDNA clones. Full-length cDNAs corresponding to

206 other members of the Flaviviridae, cloned in pBeloBAC11, have previously been 
Formatted for Veterinary Microbiology (special ESVV Pestivirus issue)

207 shown to be stable after multiple generations in E.coli cells (Yun et al., 2003;

208 Fan and Bird, 2008) and we anticipate that our constructs will display similar stability in the bacterial host but this does need to be determined.

211 DNAs from a single full-length PCR product, which gives an increased flexibility

212 in the design of new genetically modified pestiviruses. Since long RT-PCR

213 preserves the genetic variants present in a virus population (Chumakov, 1996)

214 many independent full-length cDNA clones can be obtained and tested which

215 increases the probability of finding infectious constructs. For targeted design of

216 genetically modified pestiviruses, the efforts can now be expedited and focused

217 on, in principal, any pestiviral strain and is hence not limited by the availability of

218 existing infectious cDNAs. Furthermore, our full-genome amplification protocol

219 obviates the time-consuming and costly process of construction and screening

220 of infectious clones by traditional methods. Therefore, the full-genome

221 amplification strategy significantly simplifies and streamlines the workflow for

222 the generation of new recombinant pestiviruses and also facilitates direct full-

223 length sequence analysis.

225 Acknowledgements

226 We are grateful to Gabriela Adam and Doreen Reichelt for technical assistance.

227 We thank Dr. Donata Kalthoff for her valuable advice regarding cloning in BAC

228 vectors. This work was supported by the EU Network of Excellence, EPIZONE

229 (FOOD-CT-2006-016236) and by the Danish Research Council for Technology

230 and Production Sciences (DRCTPS grant 274-07-0198). 
Formatted for Veterinary Microbiology (special ESVV Pestivirus issue)

\section{Conflict of interest statement}

232 None

233

234 References

235 Chumakov, K.M., 1996. PCR engineering of viral quasispecies: a new method

236 to preserve and manipulate genetic diversity of RNA virus populations. J. Virol.

$237 \quad 70,7331-7334$.

238 Fan, Z.C., Bird, R.C., 2008. An improved reverse genetics system for

239 generation of bovine viral diarrhea virus as a BAC cDNA. J Virol Methods 149, $240 \quad 309-315$.

241 Gritsun, T.S., Gould, E.A., 1998. Development and analysis of a tick-borne

242 encephalitis virus infectious clone using a novel and rapid strategy. J. Virol.

243 Methods 76, 109-120.

244 Jones, L.R., Zandomeni, R.O., Weber, E.L., 2006. A long distance RT-PCR

245 able to amplify the Pestivirus genome. J. Virol. Methods 134, 197-204.

246 Meyers, G., Tautz, N., Becher, P., Thiel, H.J., Kummerer, B.M., 1996. Recovery

247 of cytopathogenic and noncytopathogenic bovine viral diarrhea viruses from

248 cDNA constructs. J. Virol. 70, 8606-8613.

249 Moormann, R.J., van Gennip, H.G., Miedema, G.K., Hulst, M.M., van Rijn, P.A.,

250 1996. Infectious RNA transcribed from an engineered full-length cDNA template

251 of the genome of a pestivirus. J. Virol. 70, 763-770. 
Formatted for Veterinary Microbiology (special ESVV Pestivirus issue)

252 Oleksiewicz, M.B., Rasmussen, T.B., Normann, P., Uttenthal, A., 2003.

253 Determination of the sequence of the complete open reading frame and the

254 5'NTR of the Paderborn isolate of classical swine fever virus. Vet. Microbiol. 92, $255 \quad 311-325$.

256 Rasmussen, T.B., Reimann, I., Hoffmann, B., Depner, K., Uttenthal, A., Beer,

257 M., 2008. Direct recovery of infectious pestivirus from a full-length RT-PCR

258 amplicon. J Virol Methods 149, 330-333.

259 Rasmussen, T.B., Uttenthal, A., Reimann, I., Nielsen, J., Depner, K., Beer, M.,

2602007 . Virulence, immunogenicity and vaccine properties of a novel chimeric

261 pestivirus. J Gen Virol 88, 481-486.

262 Reimann, I., Depner, K., Trapp, S., Beer, M., 2004. An avirulent chimeric

263 Pestivirus with altered cell tropism protects pigs against lethal infection with

264 classical swine fever virus. Virology 322, 143-157.

265 Ruggli, N., Tratschin, J.D., Mittelholzer, C., Hofmann, M.A., 1996. Nucleotide

266 sequence of classical swine fever virus strain Alfort/187 and transcription of

267 infectious RNA from stably cloned full-length cDNA. J. Virol. 70, 3478-3487.

268 Tellier, R., Bukh, J., Emerson, S.U., Miller, R.H., Purcell, R.H., 1996. Long PCR

269 and its application to hepatitis viruses: amplification of hepatitis $A$, hepatitis $B$,

270 and hepatitis C virus genomes. J. Clin. Microbiol. 34, 3085-3091.

271 Vassilev, V.B., Collett, M.S., Donis, R.O., 1997. Authentic and chimeric full-

272 length genomic cDNA clones of bovine viral diarrhea virus that yield infectious 273 transcripts. J. Virol. 71, 471-478. 
Formatted for Veterinary Microbiology (special ESVV Pestivirus issue)

274 Wang, K., Boysen, C., Shizuya, H., Simon, M.I., Hood, L., 1997. Complete

275 nucleotide sequence of two generations of a bacterial artificial chromosome

276 cloning vector. Biotechniques 23, 992-994.

277 Warming, S., Costantino, N., Court DL, Jenkins, N.A., Copeland, N.G., 2005.

278 Simple and highly efficient BAC recombineering using galK selection. Nucleic

279 Acids Res. 33, e36.

280 Yun, S.I., Kim, S.Y., Rice, C.M., Lee, Y.M., 2003. Development and application

281 of a reverse genetics system for Japanese encephalitis virus. J Virol 77, 6450-

2826465.

283 Zhang, F., Huang, Q., Ma, W., Jiang, S., Fan, Y., Zhang, H., 2001. Amplification

284 and cloning of the full-length genome of Japanese encephalitis virus by a novel

285 long RT-PCR protocol in a cosmid vector. J. Virol. Methods 96, 171-182.

286

287 
Formatted for Veterinary Microbiology (special ESVV Pestivirus issue)

288 Table 1: Primers used for long RT-PCR ${ }^{a}$

289 Primers for CSFV-C and CSFV-Paderborn

290 5'Paderborn_T7 (forward)

IAATACGACTCACTATAGTATACGAGGTTAGCTCGTCCTCGTGTACAACATT

291 5'Paderborn_T7_Notl (forward)

ATATGCGGCCGCTAATACGACTCACTATAGTATACGAGGTTAGCTCGTCCTCGTGTACAACATT

292 3'Paderborn (reverse)

293 3'Paderborn-Notl (reverse)

294 3'Paderborn_cDNA (reverse) GGGCCGTTAGGAAATTACCTTAGTCCAACTGTGGA

ATATGCGGCCGC GGGCCGTTAGGAAATTACCTTAGTCCAACTGTGGA

GGGCCGTTAGGAAATTACCTTAGT

295 5'C-strain_T7 (forward)

296 5'C-strain_T7_Notl (forward)

IAATACGACTCACTATAGTATACGAGGTTAGTTCATTCTCGTATACACGATTGGACAAATC

ATATGCGGCCGCTAATACGACTCACTATAGTATACGAGGTTAGTTCATTCTCGTATACACGATTGGACAAATC

297

298 Primers for BVDV-CP7

299 5'BVDV CP7_T7 (forward)

TAATACGACTCACTATAGTATACGAGAATTAGAAAAGGCAC

$300 \quad 3$ 'BVDV CP7 (reverse)

GGGTGACGTCGGGTGTACCCTCATAC

301

302 Primers for BDV-Gifhorn

303 5'T7Gif-Notl (forward)

ATATGCGGCCGCTAATACGACTCACTATAGTATACGAGAGTAGTTCAGGCTCGTATGCAAAATTGGGTGTTTC

304 3'Gif-Notl (forward) ATATGCGGCCGCGGGGCTGTTAGGGTTTTTCCTTAATCCAACTATGGACTTCAG

305 a T7 promoter is underlined, and Notl sites are shown in italics.

$306 \quad{ }^{\mathrm{b}}$ Same primer was also used as cDNA primer. 
Formatted for Veterinary Microbiology (special ESVV Pestivirus issue)

307 Fig. 1. Full-genome amplification of pestiviruses by long RT-PCR using T7-

308 5'NTR and 3'NTR primers. The pictures show agarose gel electrophoresis of

309 the full-length amplicons of BDV-Gifhorn (A, $1 \mu \mathrm{l})$, BVDV-CP7 (B, $1 \mu \mathrm{l})$, CSFV-C

$310(\mathrm{C}, 10 \mu \mathrm{l})$ and CSFV-Paderborn $(\mathrm{D}, 10 \mu \mathrm{l})$.

311

312 Fig. 2. Full-genome amplification of pestiviruses by long RT-PCR using T7-

313 5'NTR and 3'NTR primers containing Notl restrictions sites. The picture shows

314 agarose gel electrophoresis of the full-length amplicons of BDV-Gifhorn (A, 10

$315 \mu \mathrm{l})$, CSFV-C $(\mathrm{B}, 10 \mu \mathrm{l})$ and CSFV-Paderborn $(\mathrm{C}, 10 \mu \mathrm{l})$. The non-specific bands

316 in A and C are most likely caused by the increased complexity of the primers

317 (extra Notl sites).

319 Fig. 3. Not digestion of BAC transformants. DNA preparations from BAC

320 transformants were digested with Notl and analysed by agarose gel

321 electrophoresis. Four have the correct size insert (A-C, E), whereas one (D) has

322 a smaller insert, and another $(F)$ contained no detectable BAC vector.

324 Fig. 4. Recovery of infectious CSFV-Paderborn derived from in vitro transcribed 325 pBeloPader10 RNA electroporated into porcine SK6 cells. The pictures show 326 immunofluorescence (IF) staining of cells expressing the NS3 proteins after the

$3271^{\text {st }}($ Panel $A)$ and $2^{\text {nd }}($ Panel $B)$ cell culture passage. Panels $C$ and $D$ are control 328 cells electroporated without any RNA transcripts. 
Formatted for Veterinary Microbiology (special ESVV Pestivirus issue)

332 Figure 1.

333

334
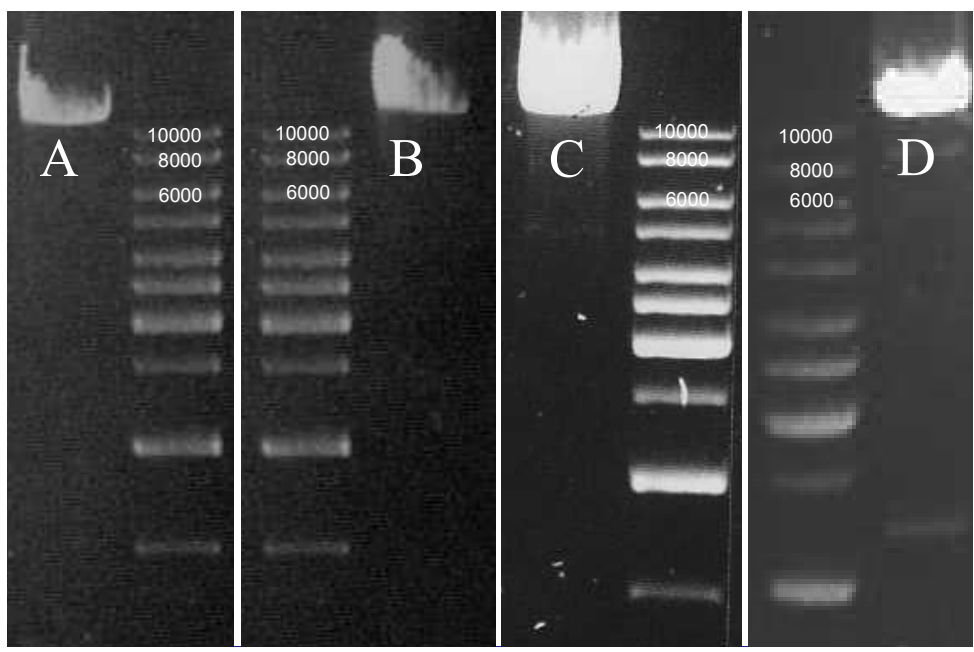

335

336 
Formatted for Veterinary Microbiology (special ESVV Pestivirus issue)

337 Figure 2.

338

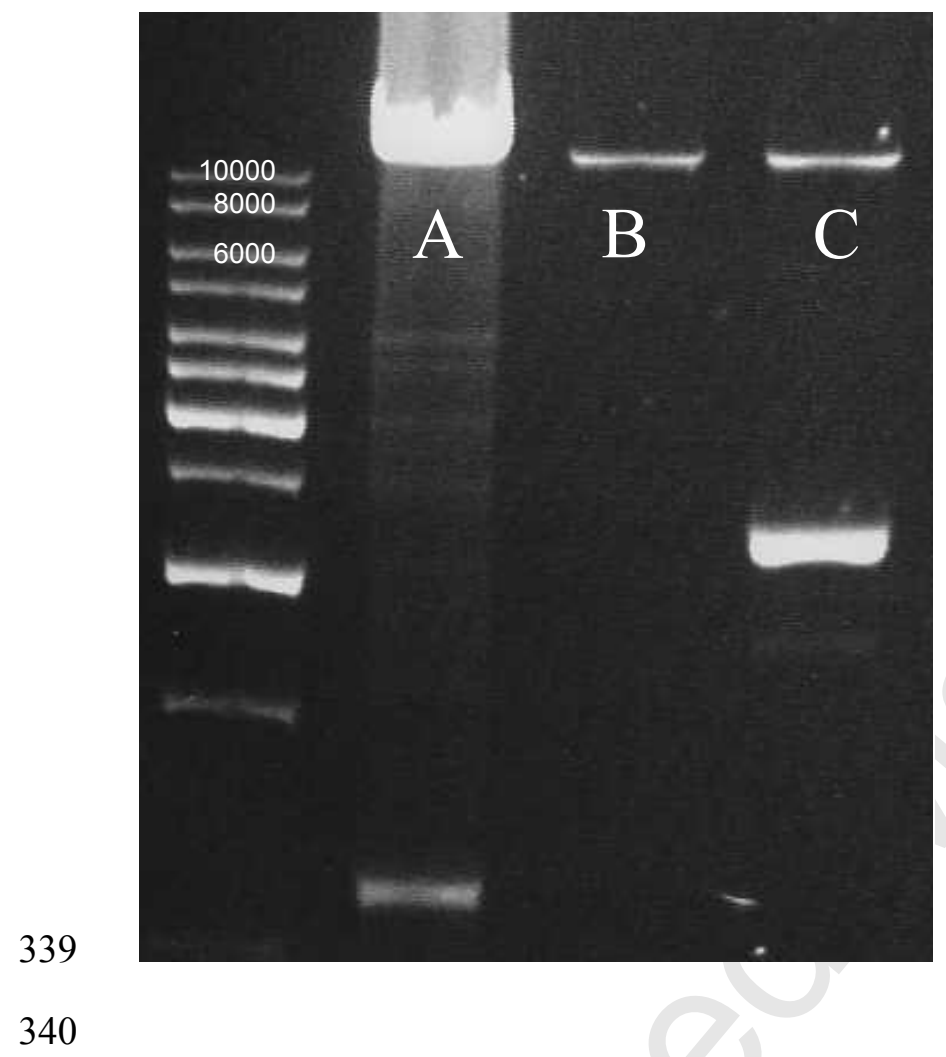


Formatted for Veterinary Microbiology (special ESVV Pestivirus issue)

341 Figure 3.

342

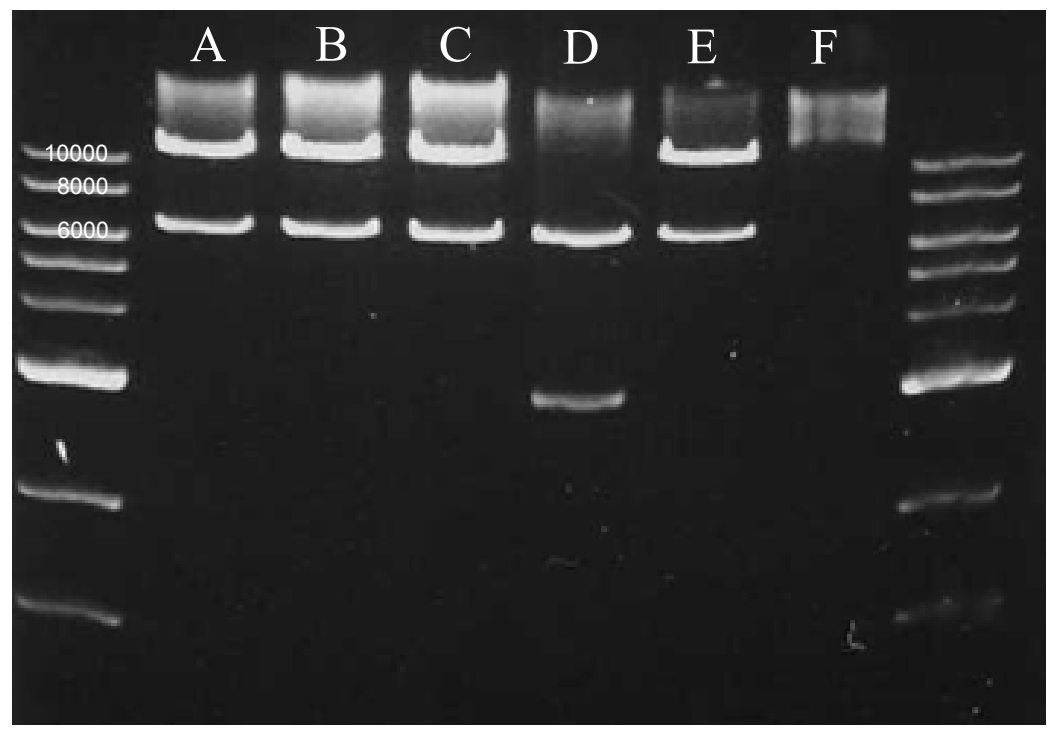

343

344 
Formatted for Veterinary Microbiology (special ESVV Pestivirus issue)

345 Figure 4.

346

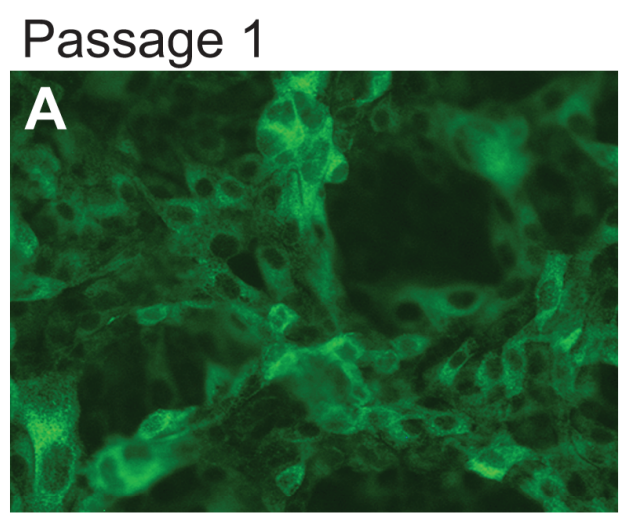

Passage 2

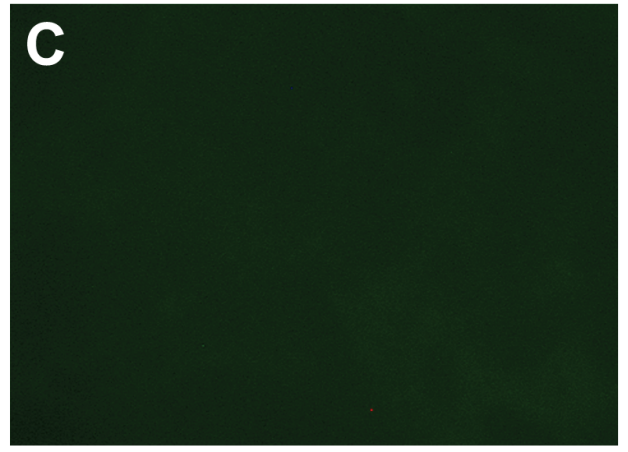

B

347

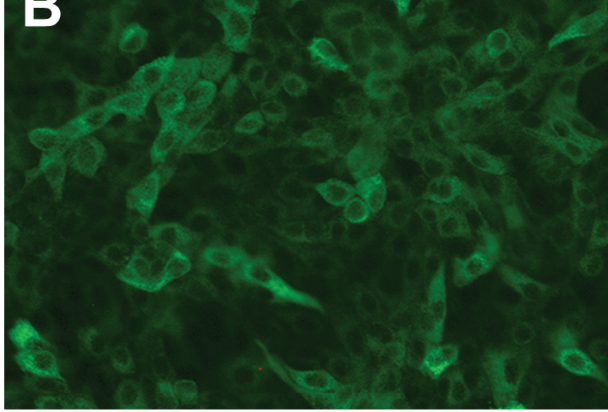

D

348 